\title{
Journal of the International AIDS

\section{Prevention of mother-to-child transmission of HIV in the HAART era: patterns in use of neonatal prophylaxis} K England

\author{
Address: UCL Institute of Child Health, London, UK \\ from Ninth International Congress on Drug Therapy in HIV Infection \\ Glasgow, UK. 9-13 November 2008 \\ Published: 10 November 2008 \\ Journal of the International AIDS Society 2008, I I (SuppI I):P2 I8 doi:I0.II86/I758-2652-I I-SI-P2 I8
}

This abstract is available from: http://www.jiasociety.org/content/II/SI/P2I8

(c) 2008 England; licensee BioMed Central Ltd.

\section{Background}

With increasing use of HAART in pregnancy, there are concerns regarding the safety of exposure to combinations of antiretroviral drugs in pregnancy and neonatal life. Clinical trial data are unavailable regarding the efficacy of different neonatal prophylactic regimens for infants born to women on HAART. There is increasing recognition of the need to balance reducing MTCT risk and minimizing fetal/neonatal ART exposure.

\section{Methods}

In the European Collaborative Study, HIV-infected pregnant women are enrolled and their infants followed in 10 European countries. Data on 2,674 mother-child pairs enrolled in the western European sites between January 1998 and June 2008 were analysed, and patterns of neonatal prophylaxis, MTCT rates, and risk of infant anaemia investigated.

\section{Summary of results}

Overall, 1,820 (68\%) women received HAART in pregnancy, $297(11 \%)$ zidovudine (ZDV) monotherapy and the remainder no antenatal ART. Of the 1,820 motherchild pairs with antenatal HAART use, the majority of infants received neonatal prophylaxis with $\mathrm{ZDV}$, although $303(17 \%)$ received a combination of two or more drugs. Of the 1,275 children with duration of ZDV prophylaxis available, 185 (14\%) received ZDV for 2 weeks (all intravenously), and the remainder received oral ZDV: 95 (7\%) for 4 weeks and 947 (74\%) for 6 weeks. Among infants born to women receiving no antenatal ART, $24(9 \%)$ received three drugs. There were trends in the type and duration of neonatal prophylaxis over the 10 -year period. The proportion of children receiving 6 weeks of ZDV decreased from $82 \%$ in $1998-2000$ to $63 \%$ after 2004 (p $<0.001$ ) while the proportion receiving ZDV for 4 weeks increased during the same periods from $3 \%$ to $19 \%$ (p < 0.001 ). Of children born to women on HAART, the proportion receiving at least two drugs decreased over the 10year period from $23 \%$ in $1998-2000$ to $16 \%$ after 2004 (p $=0.02$ ). With this decrease, the proportion of children experiencing severe anaemia during the same period halved, from $4 \%$ in $1998-2000$ to $2 \%$ after 2004 . The overall MTCT rate was 2.6\% (95\% CI 2.0-3.4\%) and $1.4 \%$ (95\% CI $0.8-2.2 \%$ ) among infants of mothers receiving HAART; in the latter group the MTCT rate was $0.9 \%$ for those receiving ZDV monotherapy and $1.6 \%$ for those on two or more drugs.

\section{Conclusion}

In the absence of evidence from clinical trials, practices for prescribing neonatal prophylaxis vary in the HAART era, with evidence of shorter durations in recent years. Prevalence of severe anaemia among infants exposed to ART in utero and early life remains low overall. 